\title{
Analysis of Binding Interaction between Captopril and Human Serum Albumin
}

\author{
Xiaoyan Gao, Yingcai Tang, Wanqi Rong, Xiaoping Zhang, Wujie Zhao, Yanqin Zi \\ Department of Chemistry and Materials Science, Huaibei Normal University, Huaibei, China \\ E-mail: ziyanqin@163.com \\ Received October 8, 2010; revised January 24, 2011; accepted May 10, 2011
}

\begin{abstract}
The interaction between captopril, an inhibitor of angiotensin converting enzyme and human serum albumin, a principal plasma protein in the liver has been investigated in vitro under a simulated physiological condition by UV-vis spectrophotometry and fluorescence spectrometry. The intrinsic fluorescence intensity of human serum albumin was strongly quenched by captopril. The binding constants and the number of binding sites can be calculated from the data obtained from fluorescence quenching experiments. The negative value of $\Delta G^{0}$ reveals that the binding process is a spontaneous process. According to the van't Hoff equation, the standard enthalpy change $\left(\Delta H^{0}\right)$ and standard entropy change $\left(\Delta S^{0}\right)$ for the reaction were calculated to be $35.98 \mathrm{KJ} \cdot \mathrm{mol}^{-1}$ and $221.25 \mathrm{~J} \cdot \mathrm{mol}^{-1} \mathrm{~K}$. It indicated that the hydrophobic interactions play a main role in the binding of captopril to human serum albumin. In addition, the distance between captopril (acceptor) and tryptophan residues of human serum albumin (donor) was estimated to be $1.05 \mathrm{~nm}$ according to the Förster's resonance energy transfer theory. The results obtained herein will be of biological significance in pharmacology and clinical medicine.
\end{abstract}

Keywords: Human Serum Albumin, Captopril, Fluorescence Quenching, Stern-Volmer Equation, The Förster's Resonance Energy Transfer Theory

\section{Introduction}

Captopril, as show in Figure 1, 1-[(2S)-3-mercapto-2methyl-1-oxopropyl]-L-proline, contains two centers of dissymmetry, one associated with the (S)-proline portion and the other associated with the 3-mercapto-2-methylpropionic acid side chain. As an inhibitor of angiotensin converting enzyme (ACEI) with a short duration of

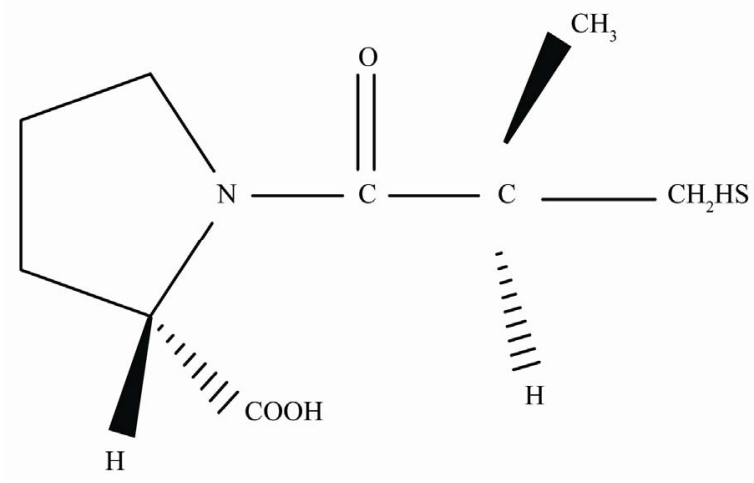

Figure 1. The structure of captopril. action [1], it is a drug with a number of cellular actions and clinical applications, and it now extensively used for treatment of chronic heart failure and hypertention [2] for it can catalyze the conversion of angiotensin I to angiotensin II. It has been shown to reduce proteinuria [3] and retard the progression of renal failure in patients with insulin dependent diabetes mellitus and nephropathy, and provides the basis of its use against hypertension. The drug stimulates prostaglandin production and release IL-2 production [4], and possesses immunosuppressant activity. Besides its vasodilative effects, it possesses inhibiting effects on hypertrophy and remodeling of myocardium without interference with the metabolism of fatty acid, and can improve the patients' quality of life [5, 6]. Some experimental studies have suggested that captopril has antiarrhythmic activity, a reduction of arrhythmias induced by isehemia, reperfusion, or digitalis toxicity [7]. Furthermore, some clinical reports have showed that captopril can decrease the frequency and grade of ventricular ectopy in patients with heart failure or cardiac infarction.

It is well known that protein is an important chemical 
substance in almost of the life and the main target of all medicines in living beings. Human serum albumin (HSA), the most abundant protein in human plasma, acts as a carrier for transporting many ligands, including fatty acids, amino acids, metal ions, and a variety of pharmaceuticals [8,9] and disposer of many endogenous and exogenous compounds $[10,15]$. It is synthesized in the liver, and exported as a non-glycosylated protein. HSA is a single polypeptide chain of 585 amino acid residues and comprises three structurally homologous domains: I (residues 1-195); II (196-383); and III (384-585). It is folded into three homologous domains each of which contains two subdomains (A and B). HSA has significant physiological function and is one of the model proteins commonly used in methodological research on immunoassay. It can interact with many endogenous and exogenous substances, and it is to bind a wide variety of endogenous and exogenous substances such as, hormones, fatty acids, bilirubin, and foreign molecules such as drugs $[16,17]$. It is important to study the interaction of drug with the protein for protein-drug binding plays an important role in pharmacology and pharmacodynamics. The information on the interaction of HSA and drug can help us better understand the absorption and distribution of the drug.

To better understand the pharmacological activities of captopril at molecular level, we characterized the interaction between captopril and HSA by different spectroscopic methods as our previous studies. Efforts were made to investigate the quenching mechanism, binding constants, binding sites, binding mode, and binding distance. Moreover, synchronous fluorescence was employed to probe conformational changes induced by captopril.

\section{Experimental}

\subsection{Apparatus and Reagents}

Absorption spectra were recorded on TU1901 UV/Vis Spectrophotometer (PGeneral, Beijing, China). An RF5301PC fluorescence spectrometer (Jasco, Japan) was applied to record the fluorescence spectra. The $\mathrm{pH}$ values were measured with a PHS-23 meter (Shanghai Secondly Analytical Instruments, China).

HSA $\left(1.0 \times 10^{-4} \mathrm{~mol} \cdot \mathrm{L}^{-1}\right)$ was prepared by dissolving $3.3250 \mathrm{~g}$ of HSA (66500 Da, Shanghai Wenhao Biochemistry Tech., Shanghai, P. R. China) in $500 \mathrm{ml}$ of deionized water. Catopril solution $\left(5.0 \times 10^{-3} \mathrm{~mol} \cdot \mathrm{L}^{-1}\right)$ was prepared by dissolving $0.2720 \mathrm{~g}$ of captopril tablets (217.29 Da, Shanghai XuDong hoop pharmaceutical Co., LTD., Shanghai, PR China) in $250 \mathrm{~mL}$ of water. The solution should be stored in a refrigerator freezer. The working solutions were obtained by diluting the stock solution with water. Britton-Robinson buffer solutions (B-R) were prepared by mixing the mixed acid (composed of $0.04 \mathrm{~mol} \cdot \mathrm{L}^{-1} \mathrm{H}_{3} \mathrm{PO}_{4}, \mathrm{HAc}$ and $\mathrm{H}_{3} \mathrm{BO}_{3}$ ) with 0.2 $\mathrm{mol} \cdot \mathrm{L}^{-1} \mathrm{NaOH}$ in proportion. The buffer solutions were prepared to adjust the acidity of the system. The $\mathrm{pH}$ value of solutions was kept at 7.24.

Deionized water was used for the preparation of some solutions. All chemicals used were of analytical-reagent or higher grade.

\subsection{General Procedure}

All studies were carried out in $10 \mathrm{~mL}$ calibrated tubes. In tubes, $1.00 \mathrm{~mL}$ known B-R buffer, $1.0 \mathrm{ml}$ of $1.0 \times 10^{-5}$ $\mathrm{mol} \cdot \mathrm{L}^{-1} \mathrm{HSA}$ solution and a known volume of the standard captopril solution were added. Then the solution was diluted to $10 \mathrm{~mL}$ with deionized water and mixed well. After reacted for 20 minutes, the solutions were taken into the optical cell.

The ultraviolet (UV) absorption spectra were measured on a TU1901 UV/Vis Spectrophotometer. The fluorescence spectra of the system were recorded on a FP6500 fluorescence spectrometer at 300-400 nm. Excitation bandwidth was $3 \mathrm{~nm}$ and emission bandwidth was 5 $\mathrm{nm}$, using a $1 \mathrm{~cm}$ quartz cell. The $\mathrm{pH}$ values were measured with a PHS-23 meter.

\section{Results and Discussion}

\subsection{Fluorescence Quenching Spectra and Quenching Mechanism of HSA by Captopril}

Any process, which decreases the fluorescence intensity of a sample, is called fluorescence quenching [18]. The basic principles like excited state reactions, molecular rearrangements, energy transfer, ground state complex formation, and collisional quenching involves in molecular interaction, which can result in quenching.

The interaction of captopril with HSA was evaluated by monitoring the intrinsic fluorescence intensity changes of HSA upon addition of captopril. The fluorescence emission spectra of HSA with various amounts of Captopril were recorded on a FP-6500 fluorescence spectrometer at $300-400 \mathrm{~nm}$ following an excitation at 280 $\mathrm{nm}$. Fluorescence quenching spectra of HSA at the presence of various concentrations of captopril are shown in Figure 2.

As seen in Figure 2, the addition of captopril led to a concentration-dependent quenching of HSA intrinsic fluorescence intensity along with a slight blue shift of maximum emission wavelength, implying that the binding of captopril to HSA occurs and the microenvironment around the chromophore of HSA is changed upon 


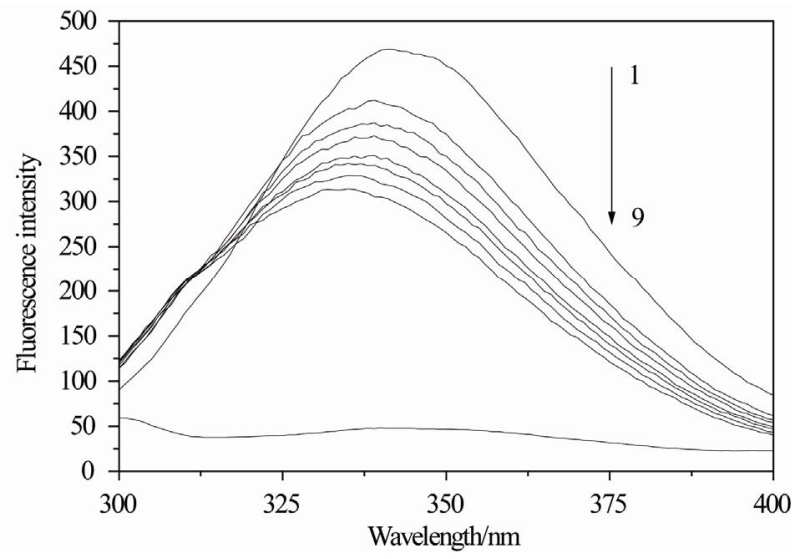

Figure 2. Fluorescence emission spectra of HSA in the absence and presence of increasing amount of captopril, $\lambda_{\mathrm{ex}}=$ $280 \mathrm{~nm}, \mathrm{C}_{\mathrm{HSA}}=1.0 \times 10^{-6} \mathrm{~mol} \cdot \mathrm{L}^{-1} ; \mathrm{C}_{\text {captopril }}=(1-8: 0.0,1.25$, $1.5,1.75,2.0,2.25,2.5$ and 2.75$) \times 10^{-4} \mathrm{~mol} \cdot \mathrm{L}^{-1}$.

addition of captopril. With the increasing concentration of captopril, the fluorescence intensity decreased gradually. Such strong quenching clearly indicated the binding of captopril with HSA.

Fluorescence quenching could proceed via different mechanisms, usually classified as dynamic quenching and static quenching. Dynamic and static quenching can be distinguished by their different dependence on temperature. Higher temperatures will result in faster diffusion and hence larger amounts of collisional quenching and higher temperatures will typically result in the dissociation of weekly bound complexes and hence smaller amounts of static quenching.

To clarify the fluorescence quenching mechanism induced by HSA, the Stern-Volmer equation Equation (1) was utilized to process the data $[19,20]$.

$$
F_{0} / F=1+K_{q} \tau_{0}[Q]=1+K_{S V}[Q]
$$

where $F_{0}$ and $F$ represent the fluorescence intensities of HSA in the absence and presence of the quencher (captopril). $[Q]$ is the concentration of the quencher, and $K_{S V}$ is the dynamic quenching constant, which is equal to $K_{q} \times \tau_{0}, K_{q}$ is the bimolecular quenching rate constant and $\tau_{0}$ is the average lifetime of the molecule without quencher. In order to confirm the quenching mechanism, the procedure of the fluorescence quenching was first assumed to be dynamic quenching. According to Equation (1), the curve of $F_{0} / F$ versus [Q] was plotted based on the experimental data. The Stern-Volmer curve was linear when the concentration of captopril ranged from 1.25 to $2.75 \times 10^{-4} \mathrm{~mol} \cdot \mathrm{L}^{-1}$ at 293.15, 300.15 and $310.15 \mathrm{~K}$ (see Figure 3). All the plots show a good linear relationship. As it is known, linear Stern- Volmer plots represent a single quenching mechanism, either static (a formation of a complex be-

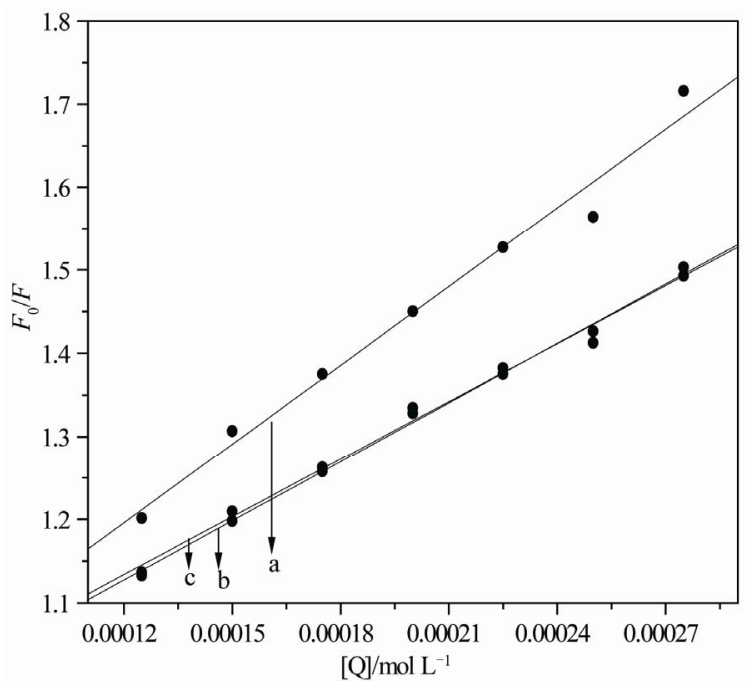

Figure 3. Plots of $F_{0} / F$ for HSA against [Q] of captopril at different temperatures: a: $293.15 \mathrm{~K}$; b: $300.15 \mathrm{~K}$; c: $310.15 \mathrm{~K}$.

tween quencher and fluorophore) or dynamic (a collisional process) [21]. In a static quenching process, generally, a linear Stern- Volmer plot indicates either only one drug binding site existing in the proximity of fluorophore or more than one binding site being all equally accessible to quenchers [22,23]. In a dynamic quenching process, the bimolecular quenching constant $K_{S V}$ is expected to increase with rising temperature because it is closely related to diffusions or diffusion coefficients.

In addition, the Stern-Volmer slope is expected to depend on the concentration of donor (HSA) in a static quenching process, whereas the slope is independent of the concentration of donor in a dynamic process. Linear fittings of the experimental data to Equation (1) afforded $K_{S V}$ and $K_{q}$ listed in Table 1. Table 1 showed that $K_{S V}$ decreases with increase in temperature. It indicates that the fluorescence quenching of HSA by captopril appears to occur via a dynamic quenching mechanism. $K_{q}$ in Table 1 was calculated by $K_{q}=K_{S V} / \tau_{0}$, and generally the values of $\tau_{0}$ for biopolymers were given as $10^{-8} \mathrm{~s}^{-1}$ [24]. However, $K_{q}$ in Table 1 is of the magnitude of $10^{11} \mathrm{~L}$ $\mathrm{mol}^{-1} \cdot \mathrm{s}^{-1}$, which is greater than the maximum diffusion collision quenching rate constant $\left(2.0 \times 10^{10} \mathrm{~L} \cdot \mathrm{mol}^{-1} \cdot \mathrm{s}^{-1}\right)$ for a variety of quenchers with biopolymer. Therefore, it suggests that the fluorescence quenching process of HSA may be mainly governed by a static quenching mechanism arising from a complex formation rather than a dynamic quenching mechanism [25].

\subsection{Binding Constant and Number of Binding Site}

In a static quenching process, small molecules will be 
Table 1. Stern-Volmer quenching constants for the captopril-HSA system at pH 7.24 (293.15 K to 310.15 K).

\begin{tabular}{cccc}
\hline $\mathrm{T}(\mathrm{K})$ & $K_{s v}\left(\times 10^{3} \mathrm{~L} \cdot \mathrm{mol}^{-1}\right)$ & $R$ & $K q\left(\times 10^{11} \mathrm{~L} \cdot \mathrm{mol}^{-1} \cdot \mathrm{s}^{-1}\right)$ \\
\hline 293.15 & 3.157 & 0.991 & 3.157 \\
300.15 & 2.370 & 0.998 & 2.370 \\
310.15 & 2.314 & 0.996 & 2.314 \\
\hline
\end{tabular}

independently bound to a set of equivalent sites on a macromolecule. Thus, the equilibrium between free and bound molecules is given by References [26,27]. When small molecules were bound independently to a set of equivalent sites on a macromolecule, theequilibrium between free and bound molecules was given by Equation (2) $[28]$.

$$
\log \left(F_{0}-F\right) / F=\log K_{b}+n \log [Q]
$$

where $K_{b}$ was the binding constant and $n$ was the number of binding sites. For the captopril-HSA system in the lower concentration range, the values for $K_{b}$ and $n$ at different temperatures can be derived from the intercept and slope of Figure 4 based on Equation (2) and presented in Table 2. Linear regression Equations (4-6) at 290.15, 300.15 and $310.15 \mathrm{~K}$ are expressed as follows:

$$
\begin{array}{lll}
\log \left(F_{0}-F\right) / F=5.1399+1.57 \times \log [Q] & 293.15 \mathrm{~K} & (3) \\
\log \left(F_{0}-F\right) / F=5.2989+1.62 \times \log [Q] & 300.15 \mathrm{~K} & (4) \\
\log \left(F_{0}-F\right) / F=5.4969+1.49 \times \log [Q] & 310.15 \mathrm{~K} & (5)
\end{array}
$$

respectively. $K_{b}$ in Table 2 shows that there exist a strong interaction between captopril and HSA and a complex formation of captopril with HSA. Furthermore, it can be inferred from the values of $\mathrm{n}$ that there is an independent class of binding sites on HSA for captopril. Otherwise, it appears that the binding constants and the number of binding sites increased with increase in temperature $[29,30]$. This may be attributed to that the capacity of captopril binding to the fact that HSA is increased with increase in temperature.

\subsection{Mode of Binding from Thermodynamic Parameters}

Intermolecular interacting forces between a small mole-

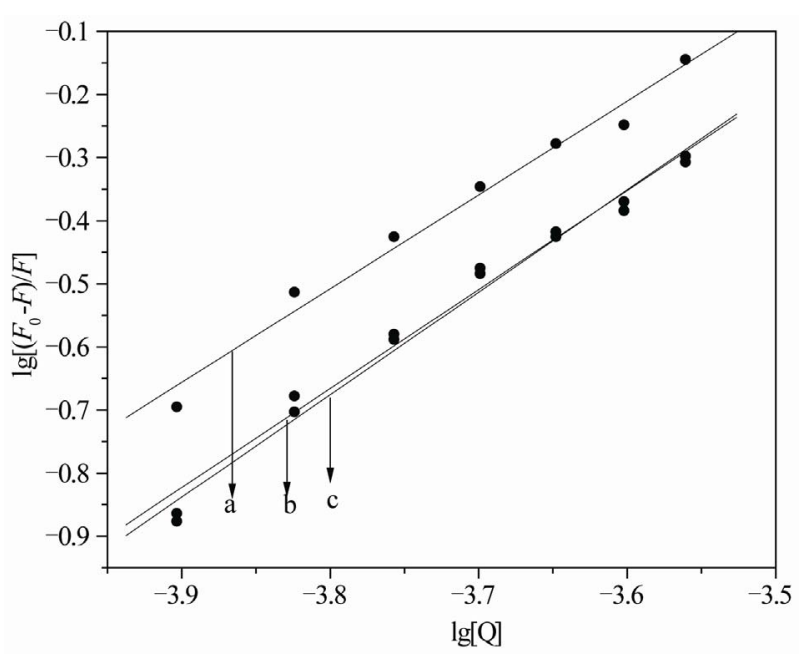

Figure 4. Double-lg plot of captopril quenching effect on the fluorescence of HSA at different temperatures: a $293.15 \mathrm{~K}$; b $300.15 \mathrm{~K}$; c 310.15 K.

cule and a biomacromolecule include hydrogen bond, van der Waals force, electrostatic and hydrophobic interactions, etc. Because of the $-\mathrm{COOH}$ and $-\mathrm{SH}$ in the molecular of Captopril, these active groups can interact with the HSA. Thermodynamic parameters for a binding interaction can be used as a major evidence to learn the nature of intermolecular forces. Thus, the temperaturedependent thermodynamic parameters for the captoprilHSA system are used to characterize the intermolecular forces between captopril and HSA.

Thermodynamic parameters such as free energy $\left(\Delta G^{0}\right)$, enthalpy $\left(\Delta H^{0}\right)$ and entropy $\left(\Delta S^{0}\right)$ due to complex formation provide an insight into the binding mode. If an enthalpy change $\left(\Delta H^{0}\right)$ does not vary significantly with temperature, its value and that for an entropy change $\left(\Delta S^{0}\right)$ can be determined by the van't Hoff equation as follows:

$$
\ln K=-\Delta H^{0} / R T+\Delta S^{0} / R
$$

Consequently, a free energy change $\left(\Delta G^{0}\right)$ for a binding interaction at different temperatures can be evaluated:

$$
\Delta G^{0}=\Delta H^{0}-T \Delta S^{0}
$$

where $K$ is the binding constant and $R$ the gas constant. The values of $\Delta G^{0}, \Delta H^{0}, \Delta S^{0}$ for captopril binding to HSA are listed in Table 2. The negative value of $\Delta G^{0}$

Table 2. Biding constants and thermodynamic parameters of captopril with HSA at different temperatures (293.15 K to $310.15 \mathrm{~K})$.

\begin{tabular}{ccccccc}
\hline$T(\mathrm{~K})$ & $K_{b}\left(\times 10^{5} \mathrm{~L} \cdot \mathrm{mol}^{-1}\right)$ & $n$ & $R$ & $\Delta H^{0}\left(\mathrm{KJ}^{\mathrm{mol}}{ }^{-1}\right)$ & $\Delta G^{0}\left(\mathrm{KJ}^{2} \cdot \mathrm{mol}^{-1}\right)$ & $\Delta S^{0}\left(\mathrm{~J} \cdot \mathrm{mol}^{-1} \cdot \mathrm{k}^{-1}\right)$ \\
\hline 293.15 & 1.38 & 1.49 & 0.992 & 35.97 & -28.84 & 221.22 \\
300.15 & 1.99 & 1.57 & 0.992 & 35.97 & -30.45 & 221.22 \\
310.15 & 3.14 & 1.62 & 0.991 & 35.97 & -32.64 & 221.22 \\
\hline
\end{tabular}


reveals that the binding process is a spontaneous process. The positive value of $\Delta S^{0}$ change arises from water molecules arranged more random around HSA and drug, caused by hydrophobic interactions between HSA and drug molecules. Besides, the positive $\Delta H^{0}$ is considered as another evidence for hydrophobic interactions. Thus, positive values for both $\Delta H^{0}$ and $\Delta S^{0}$ indicate hydrophobic interactions playing a major role in captopril binding to HSA [31].

\subsection{Conformational Changes Investigated by Synchronous Fluorescence}

Synchronous fluorescence is a very useful tool to investigate the microenvironments around the fluorophore functional groups. It is well known that the fluorescence of HSA arises from the tyrosine, tryptophan and phenylalanine residues. The change in HSA conformation upon addition of captopril can be also demonstrated by synchronous fluorescence spectra. As is known, synchronous fluorescence spectra show tyrosine residues of HSA only at the wavelength interval $(\Delta \lambda)$ of $15 \mathrm{~nm}$ and tryptophan residues of HSA only at $\Delta \lambda$ of $60 \mathrm{~nm}$. The effect of captopril on synchronous fluorescence spectra was shown in Figure 5. The synchronous spectra of captopril-HSA system were scanned at $\Delta \lambda=60 \mathrm{~nm}$ (Figure 5(a)) and $\Delta \lambda=15 \mathrm{~nm}$ (Figure 5(b)). The fluorescence of tryptophan (Figure 5(a)) was strong, with addition of captopril the fluorescence intensity decreased and no apparent shift occurred in Figure 5(a). It can be seen the fluorescence spectra of tyrosine (Figure 5(b)) was weak and addition of captopril resulted in a decrease in intensity and also no shift of maximum emission wavelength [32]. It indicated that the interaction of captopril with HSA does not significantly affect the conformation of tryptophan and tyrosine residue microregions.

\subsection{Energy Transform between Captopril and HSA}

In order to determine the precise location of captopril in HSA, the efficiency of energy transfer was studied according to the Förster resonance energy transfer theory [33]. The energy transfer was calculated by determination the emission fluorescence of donor and the UV spectra of accepter. The fluorescence quenching of HSA after binding with captopril indicated the transfer of energy between captopril and HSA has occurred. The efficient ligand-protein interaction gives rise to energy, from which the distance between two interacting molecules can be easily evaluated. The efficiency of energy transfer E, is described by the following equation (8) [34, 35]:

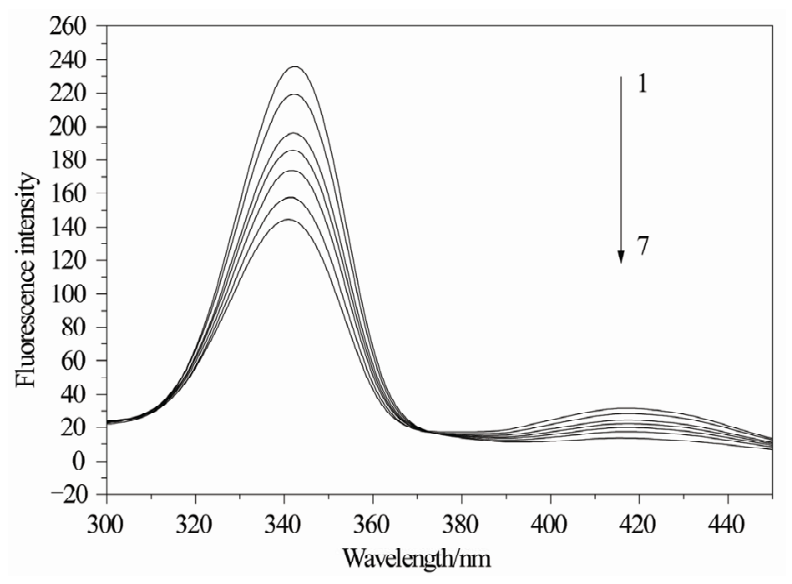

(a)

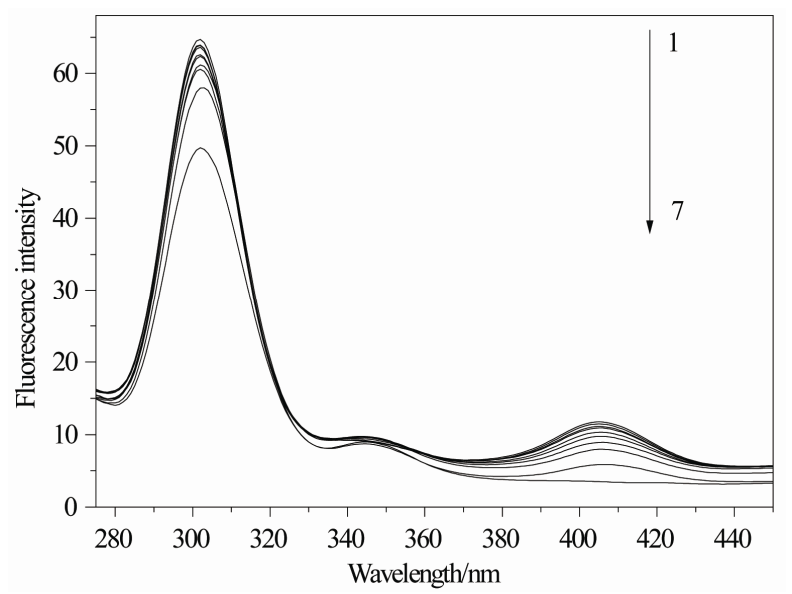

(b)

Figure 5. Synchronous fluorescence spectra of HSA in the absence andpresence of increasing amount of captopril. (a): $\Delta \lambda=60 \mathrm{~nm} ;(\mathrm{b}): \Delta \lambda=15 \mathrm{~nm}, \mathrm{~T}=300.15 \mathrm{~K}, \mathrm{pH}=7.24, \mathrm{C}_{\mathrm{HSA}}$ $=1.0 \times 10^{-6} \mathrm{~mol} \cdot \mathrm{L}^{-1}, \mathrm{C}_{\text {captopril }}=(1-7: 0.0,1.25,1.5,1.75,2.0$, 2.25 and 2.5$) \times 10^{-4} \mathrm{~mol} \cdot \mathrm{L}^{-1}$, respectively.

$$
E=1-F / F_{0}=R_{0}^{6} /\left(R_{0}^{6}+R^{6}\right)
$$

where $F_{0}$ and $F$ are the fluorescence intensity of donor in the absence and presence of acceptor, respectively, $R$ is the distance between acceptor and donor and $R_{0}$ is the critical distance, and the value of $R_{0}$ is calculated by following equation:

$$
R_{0}^{6}=8.8 \times 10^{-25} K^{2} N^{-4} \Phi J
$$

where $K^{2}$ is the spatial orientation factor of the dipole, $N$ is the refractive index of the medium, $\Phi$ is the fluorescence quantum yield of the donor, $J$ is the overlap integral of the fluorescence emission spectrum of the donor and the absorption spectrum of the acceptor. $J$ is given by

$$
J=\int_{0}^{\infty} F(\lambda) \varepsilon(\lambda) \lambda^{4} \mathrm{~d} \lambda / \int_{0}^{\infty} F(\lambda) \mathrm{d} \lambda
$$


In this equation, $F(\lambda)$ is the fluorescence intensity of the fluorescent donor of wavelength, $\lambda, \varepsilon(\lambda)$ is the molar absorption coefficient of the acceptor at wavelength. It was reported earlier that $K^{2}=2 / 3, N=1.336$, and $\Phi=$ 0.118 [36]. The overlap of UV absorbance spectrum of captopril with fluorescence spectrum of HSA was shown in Figure 6. From the above relationships, for the captopril-HSA, the values for $E$, and $R$ evaluated are the following: $J=1.09 \times 10^{-18} \mathrm{~cm}^{3} \cdot \mathrm{L} \cdot \mathrm{mol}^{-1}, R_{0}^{6}=2.369 \times$ $10^{-43} \mathrm{~cm}^{6}, E=0.15$, and $R=1.05 \mathrm{~nm}$ for HSA. The distance $R<8 \mathrm{~nm}$ [37] between donor and acceptor indicates that the energy transfer from HSA to captopril occurred with high possibility. This accord with the conditions of Förster's energy transfer theory indicated the static quenching interaction between captopril and HSA.

\section{Conclusions}

The interaction between captopril and HSA has been investigated by using fluorescence and ultraviolet (UV) absorption spectra in vitro under a simulated physiological condition $(\mathrm{pH}=7.24)$ in this work. The experimental results suggested that the fluorescence of HSA was quenched through static mode, and the pharmaceutical (captopril) can strongly binding to HSA. The synchronous fluorescence spectra showed no significant change in the conformation of HSA upon addition of captopril under experimental conditions; the binding distance $\mathrm{R}$ of $1.05 \mathrm{~nm}$ between captopril and HSA indicated that the energy transfer from HSA to captopril occurred with high possibility. In addition, the thermodynamic parameter elucidated the binding reaction was mainly driven by hydrophobic interaction.

The determinations performed herein may provide an approach to evaluate the toxic effects of chemicals on target proteins and the molecular mechanism of toxicity.

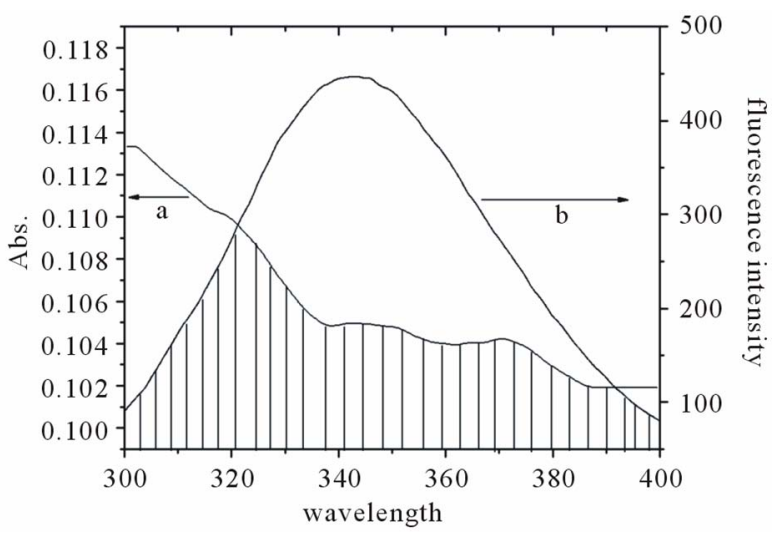

Figure 6. The overlap of the UV absorption spectrum of captopril (a) with the fluorescence emission spectrum of HAS (b) when the molar ratio is 1:1, $C_{\mathrm{HSA}}=\mathrm{C}_{\text {Captopril }}=1.0 \times$ $10^{-6} \mathrm{~mol} \cdot \mathrm{L}^{-1}, \mathrm{~T}=300.15 \mathrm{~K}, \mathrm{pH}=7.24$.
The results obtained herein will be of biological significance in pharmacology and clinical medicine.

\section{Acknowledgements}

We thank the National Key Technology R\&D Program of China (No.2008BAJ08B13) for financially supporting this work.

\section{References}

[1] A. Gupta, S. K. Prajapati, M. Singh and M. Balamurugan, "Proniosomal Powder of Captopril: Formulation and Evaluation," Molecular Pharmaceutics, Vol. 4, No. 4, May 2007, pp. 596-599. doi:10.1021/mp0700110

[2] J. K. Wang, L. Gao and Y. Liu, "Solubility of Captopril in 2-Propanol, Acetone, Acetonitrile, Methyl Acetate, Ethyl Acetate, and Butyl Acetate," Journal of Chemical \& Engineering Data, Vol. 55, No. 2, October 2010, pp. 966-967. doi:10.1021/je900401z

[3] L. S. Milner, M. Yuskiw, A. Sadeghi-Nejad and M. A. Linshaw, "Captopril Reduces the Proteinuric Effect of Human Growth Hormone in Adriamycin Nephrosis," Pediatric Nephrology, Vol. 13, No. 5, June 1999, pp. 391395. doi: $10.1007 / \mathrm{s} 004670050628$

[4] G. R. Jan Elferink and M. Ben de Koster, "Modulation of Neutrophil Migration by Captopril, Naunyn-Schmiedeberg's Arch," Pharmacol, Vol. 347, No. 5, May 1993, pp. 562-567. doi:10.1007/BF00166751

[5] Y. G. Wang and Z. Y. Lu, "Effect of Captopril on Membrane Currents of Ventricular Myocytes," Journal of Tongji Medical University, Vol. 15, No. 4, May 1995, pp. 209-211. doi:10.1007/BF02887946

[6] C. Ribuot and L. Rochette, "Converting Enzyme Inhibitors (Captopril, Enalapril, Perindopril) Prevent Early-Post Infarction Ventricular Fibrillation in The Anaesthetized Rat," Cardiovascular Drugs and Therapy, Vol. 1, No. 1, January 1987, pp. 51-55. doi:10.1007/BF02125833

[7] A. Karimia and N. Alizadehb, "Rapid Analysis of Captopril in Human Plasma and Pharmaceutical Preparations by Headspace Solid Phase Microextraction based on Polypyrrole Film Coupled to Ion Mobility Spectrometry," Talanta, Vol. 79, No. 2, July 2009, pp. 479-485. doi:10.1016/j.talanta.2009.04.016

[8] F. L. Cui, Y. R. Cui, H. X. Luo, X. J. Yao, J. Fan and Y. $\mathrm{Lu}$, "Interaction of APT with BSA or HAS," Chinese Science Bulletin, Vol. 51, No. 18, September 2006, pp. 2201-2207. doi:10.1007/s11434-006-2108-y

[9] A. K. Shaw and S. K. Pal, "Spectroscopic Studies on the Effect of Temperature on pH-Induced Folded States of Human Serum Albumin," Journal of Photochemistry and Photobiology B: Biology, Vol. 90, No. 1, January 2008, pp. 69-77.

[10] Z. Lu, Y. Zhang, H. Liu, J. Yuan, Z. Zheng and G. Zou, "Transport of a Cancer Chemopreventive Polyphenol, Resveratrol: Interaction with Serum Albumin and Hemoglobin," Journal of Fluorescence, Vol. 17, No. 5, Sep- 
tember 2007 , pp. $580-587$. doi:10.1007/s10895-007-0220-2

[11] Y. J. Hu, Y. Liu and L. X. Zhang, "Study of Interaction between Colchicines and Bovine Serum Albumin by Fluorescence Quenching Method," Journal of Molecular Structure, Vol. 750, No. 1-3, August 2005, pp. 174-178. doi:10.1016/j.molstruc.2005.04.032

[12] C. Dufour and O. Dangles, "Flavonoid-Serum Albumin Complexation: Determination of Binding Constants and Binding Sites by Fluorescence Spectroscopy," Biochimica et Biophysica Acta, Vol. 1721, No. 1-3, January 2005, pp. 164-173.

[13] M. X. Xie, M. Long, Y. Liu, C. Qin and Y. D. Wang, "Characterization of the Interaction between Human Serum Albumin and Morin," Biochimica et Biophysica Acta, Vol. 1760, No. 8, August 2006, pp. 1184-1191.

[14] H. G. Mahesha, S. A. Sighn, N. Srinivasan and R. G. Appu, "A Spectroscopic Study of the Interaction of Isoflavones with Human Serum Albumin," Febs Journal, Vol. 273, No. 3, February 2006, pp. 451-467. doi:10.1111/j.1742-4658.2005.05071.x

[15] I. Petitpas, A. A. Bhattacharya, S. Twine, M. East and S. Curry, "Crystal Structure Analysis of Warfarin Binding to Human Serum Albumin-Anatomy of Drug Site I," The Journal of Biological Chemistry, Vol. 276, No. 25, June 2001, pp. 22804-22809. doi:10.1074/jbc.M100575200

[16] F. Moreno, J. Gonza'lez-Jime'nez, "Binding of the Promen Fluorescent Probe to Human Serum Albumin: A Fluorescence Spectroscopic Study," Chemico-Biological Interactions, Vol. 121, No. 3, August 1999, pp. 237-252. doi:10.1016/S0009-2797(99)00111-8

[17] K. Yamasaki, T. Maruyama, U. Kragh-Hansen and M. Otagiri, "Characterization of Site I on Human Serum Albumin: Concept about the Structure of a Drug Binding Site," Biochimica et Biophysica Acta, Vol. 1295, No. 2, July 1996, pp. 147-157. doi:10.1016/0167-4838(96)00013-1

[18] J. R. Lakowicz, "Principles of Fluorescence Spectroscopy," 2nd Edition, Plenum Press, New York, 1999.

[19] O. R. Quayle, "The Parachors of Organic Compounds. In: an Interpretation and Catalogue," Chemical Reviews, Vol. 53, No. 3, December 1953, pp. 439-589. doi:10.1021/cr60166a003

[20] J. R. Lakowicz, "Principles of Fluorescence Spectroscopy," Plenum Press, New York, 1983.

[21] Y, B. Huang, B. Z. Liu, Z. Yu, X. Y. Gao and Y. Q. Zi, "Luminescence Quenching Effect for the Interaction of Prulifloxacin with Trypsin-Britton-Robinson Buffer Solution System," Journal of Luminescence, Vol. 130, No. 3, March 2010, pp. 360-364

[22] H. W. Zhao, M. Ge, Z. X .Zhang, W. F. Wang and G. Z. $\mathrm{Wu}$, "Spectroscopic Studies on the Interaction between Riboflavin and Albumins. Spectrochim," Acta Part A, Vol. 65, No. 3-4, November 2006, pp. 811-817. doi:10.1016/j.saa.2005.12.038

[23] J. H. Tang, S. D. Qi and X. G. Chen, "Spectroscopic Studies of the Interaction of Anti-Coagulant Rodenticide
Diphacinone with Human Serum Albumin," Journal of Molecular Structure, Vol. 779, No. 1-3, November 2005, pp. 87-95. doi:10.1016/j.molstruc.2005.07.023

[24] J. R. Lakowicz and G. Weber, "Quenching of Fluorescence by Oxygen, Probe for Structural Fluctuations in Macromolecules," Biochemistry, Vol. 12, No. 21, October 1973, pp. 4161-4170. doi:10.1021/bi00745a020

[25] W. R. Ware, "Oxygen Quenching of Fluorescence in Solution: An Experimental Study of the Diffusion Process," The Journal of Physical Chemistry, Vol. 66, No. 3, March 1962, pp. 455-458. doi:10.1021/j100809a020

[26] X. Z. Feng, Z. Lin, L. J. Yang, C. Wang and C. L. Bai, "Investigation of the Interaction between Acridine Orange and Bovine Serum Albumin," Talanta, Vol. 47, No. 5, December 1998, pp. 1223-1229.

[27] M. Jiang, M. X. Xie, Y. Liu, X. Y. Li and X. Chen, "Spectroscopic Studies on the Interaction of Cinnamic Acid and Its Hydroxyl Derivatives with Human Serum Albumin," Journal of Molecular Structure, Vol. 692, No. 1-3, April 2004, pp. 71-80. doi:10.1016/j.molstruc.2004.01.003

[28] M. X. Xie, X. Y. Xu and Y. D. Wang, "Interaction between Hesperetin and Human Serum Albumin Revealed by Spectroscopic Methods," Biochimica et Biophysica Acta, Vol. 1724, No. 1-2, June 2005, pp. 215-224.

[29] P. B. Kandagal, S. Ashoka, J. Seetharamappa, S. T. Shaikh, Y. Jadegoud and O. B. Ijare, "Study of the Interaction of an Anticancer Drug with Human and Bovine Serum Albumin: Spectroscopic Approach," Journal of Pharmaceutical and Biomedical Analysis, Vol. 41, No. 2, May 2006, pp. 393-399. doi:10.1016/j.jpba.2005.11.037

[30] Y. L. Wei, J. Q. Li, C. Dong, S. Shuang, D. S. Liu and C. W. Hui, "Investigation of the Association Behaviors between Biliverdin and Bovine Serum Albumin by Fluorescence Spectroscopy," Talanta, Vol. 70, No. 2, September 2006, pp. 377-382. doi:10.1016/i.talanta.2006.02.052

[31] P. D. Ross and S. Subramanian, "Thermodynamics of Protein Association Reactions: Forces Contributing to Stability," Biochemistry, Vol. 20, No. 11, May 1981, pp. 3096-3210. doi:10.1021/bi00514a017

[32] T. Förster, "Modern Quantum Chemistry," In: O. Sinaoglu, Ed., Academic Press, New York, Vol. 3, 1965.

[33] G. Cristobal, R. Dos and D. M. Pierre, "Fluorescence Resonance Energy Transfer Spectroscopy is a Reliable "Ruler" for Measuring Structural Changes in Proteins: Dispelling the Problem of the Unknown Orientation Factor," Journal of Structural Biology, Vol. 115, No. 2, September 1995, pp. 175-185. doi:10.1006/jsbi.1995.1042

[34] D. C. Saha, K. Ray and T. N. Misra, "Energy Transfer in Triton-X 100 Micelles: A Fluorescence Study," Spectrochimica Acta Part A, Vol. 56, No. 4, March 2000, pp 797-801. doi:10.1016/S1386-1425(99)00169-9

[35] C. Bertucci and E. Domenici, "Reversible and Covalent Binding of Drugs to Human Serum Albumin: Methodological Approaches and Physiological Relevance," Current Medicinal Chemistry, Vol. 9, No. 15, August 2002, pp. 1463-1481. 
[36] B. Valeur and J. C. Brochon, "New Trends in Fluorescence Spectroscopy," 6th Edition, Springer Press, Berlin, 1999.

[37] G. Z. Chen, X. Z. Huang, J. G. Xu, Z. X. Zheng and Z. B.
Wang, "The Methods of Fluorescence Analysis (in Chinese)," 2nd Edition, Science in China Press, Beijing, 1990. 